\title{
ORIGINAL ARTICLE \\ Potential for enhanced therapeutic activity of biological cancer therapies with doxycycline combination
}

\author{
H Tang ${ }^{1,2}$, P Sampath ${ }^{1}$, X Yan ${ }^{2}$ and SH Thorne ${ }^{1}$
}

Despite significant strides made in the clinical translation of adoptive immune cell therapies, it is apparent that many tumors incorporate strategies to avoid recognition by receptors expressed on the immune cells, such as NKG2D. Strategies that stabilize the expression of ligands for these receptors may enhance the therapeutic potential of these and related therapies. Doxycycline inhibits matrix metalloproteinases (MMPs) that act to cleave the extracellular domain of MICA/B, ligands for the NKG2D receptor. Doxycycline treatment blocked shedding of MICA/B from a panel of human tumor cells, but also acted to increase their expression and cell surface translocation, possibly through its action on ATM. This meant that many tumor cells displayed increased MICA/B expression and enhanced susceptibility to CIK cells. Interestingly, doxycycline also selectively enhanced the replication of oncolytic vaccinia in many tumor cell lines, leading to increased sensitivity to these therapies. Combination (CIK-oncolytic vaccinia) therapies used in conjunction with doxycycline led to increased anti-tumor effects. The unexpected and pleiotropic beneficial anti-tumor effects of doxycycline on both immune cell and oncolytic viral therapies make it an excellent candidate for rapid clinical testing.

Gene Therapy (2013) 20, 770-778; doi:10.1038/gt.2012.96; published online 3 January 2013

Keywords: doxycycline; NKG2D; immunotherapy; oncolytic virus; MICA

\section{INTRODUCTION}

Adoptively transferred immune cells have made significant clinical strides in recent years in the treatment of multiple cancer types. ${ }^{1-5}$ However several limitations remain, and approaches that increase either the targeting or the potency of these immune cells are needed.

One such approach that we have previously described uses some immune cell populations (including Cytokine Induced Killer, CIK cells ${ }^{6,7}$ ) as carrier vehicles to deliver oncolytic viruses to tumors, so increasing their potency. ${ }^{8,9}$ Oncolytic viruses are viral therapies whose replication is restricted to tumor cells, ${ }^{10}$ and strains of engineered vaccinia virus have displayed encouraging pre-clinical $^{11,12}$ and clinical responses. ${ }^{13-15}$ The capacity to utilize CIK cells as delivery vehicles significantly enhances their therapeutic capabilities.

CIK cells, like Lymphokine Activated Killer (LAK), ${ }^{16,17}$ and some experimental cell lines (TALL-104, ${ }^{18}$ NK-92 ${ }^{19}$ ) express the receptor NKG2D on their surface that recognizes stress response ligands, such as MICA and MICB that are frequently expressed on cancer cells. ${ }^{20}$ However many tumors have also developed strategies to evade detection by these and related therapies. In particular NKG2D ligands may be cleaved from the surface of cancer cells, ${ }^{21,22}$ or their expression may be down regulated. ${ }^{23}$ Strategies that increase and stabilize NKG2D-ligand expression on tumor cells would therefore increase the potency of many immune cell therapies.

It has been found that some histone deacetylase inhibitors can upregulate expression of $\mathrm{MICA} / \mathrm{B}^{24-26}$ however this frequently leads to further increased shedding of the extracellular domains of these surface proteins. Shedding of soluble MICA/B is typically mediated by the action of matrix metalloproteinases (MMP). ${ }^{22}$ Although MMP inhibitors can block this shedding, ${ }^{24}$ no targeted
MMPi are currently approved for clinical use. ${ }^{27}$ Because the commonly used antibiotic doxycycline has known MMPi activity, ${ }^{28}$ it was decided to test the applicability of using doxycycline in combination with CIK cell therapy. However, the pleiotropic functions of tetracyclines such as doxycycline ${ }^{29}$ meant that a thorough examination of the effects of this treatment on CIK cell therapy and CIK cell-oncolytic vaccinia virus combination therapy was required. Especially as we observed that doxycycline apparently enhanced vaccinia replication in at least some cell types.

Initial experiments determined that doxycycline treatment not only reduced the shedding of sMICA/B, it actually enhanced the levels of surface expression of MICA and MICB on tumor cells through multiple mechanisms, leading to greatly stabilized surface expression across many cell lines. This observation, coupled with the observation that doxycycline also increased vaccinia replication in tumor cells led us to try to define the mechanisms behind these effects and to determine the effects of doxycycline treatment on anti-tumor activity of these therapies used alone or in combination in different mouse models.

\section{RESULTS}

Doxycycline treatment reduces shedding of soluble MICA and MICB and increases surface expression in cancer cells

A panel of 12 human tumor cell lines, (primarily ovarian, colorectal and breast cancers) and several non-tumor cell lines were grown in culture and the levels of soluble MICA and MICB released into the media after $24 \mathrm{~h}$, were quantified by ELISA. Only 4 cell lines released detectable amounts of soluble MICA/B under these conditions (HeLa; UCl-101; UCI-107 and MDA-MB-231). The effects of exposing these cells to a pan MMP inhibitor or doxycycline for

${ }^{1}$ University of Pittsburgh Cancer Institute, Departments of Surgery and Immunology, University of Pittsburgh, Pittsburgh, PA, USA and ${ }^{2}$ Institute of Clinical and Basic Medical Sciences, The First People's Hospital of Yunnan Province (The Kunhua Affiliated Hospital of Kunming University of Science and Technology), Kunming, Yunnan, China. Correspondence: Dr SH Thorne, 1.46e Hillman Cancer Center, University of Pittsburgh, 5117 Centre Avenue, Pittsburgh, PA-15213, USA. 
this period was examined (Figure 1a). In most cases either treatment significantly reduced shedding $(P<0.05)$, typically by $2-5$-fold. There was only one cell line where only one of the treatments reduced shedding of either SMICA or SMICB; for SMICB release from $\mathrm{UCl}-101$, where only $\mathrm{MMPi}$ reduced shedding significantly (HeLa cells displayed very low shedding levels $(<25 \mathrm{pg} / \mathrm{ml} / 24 \mathrm{~h})$ and neither treatment had any significant effect). Combining both treatments did not produce additional benefits (data not shown).

Further, when the overall level of MICA/B on the surface of all 13 cell lines were assayed by flow cytometry (Figure 1b), doxycycline was found to significantly increase the level of surface MICA/B expression both on those cell lines found to shed the ligands (UCl-101; UCl-107; MDA-MB-231), as well as other cell lines that did not shed, and in which MMPi had no effect (Ovcar4, DLD1, MCF-7). The only cell lines in which doxycycline had no effect were those with very low ( $<10 \%$ ) background level of MICA/B (Skov3; Ovcar8, HT-29; H596) and in the non-tumor cell line MRC-5. It therefore appears that doxycycline is able to stabilize MICA/B surface expression through additional mechanisms beyond inhibition of MMPs. Histone deacetylase inhibitors (HDACi) are also known to enhance MICA/B surface expression levels, and so a panel of HDACi (Trichostatin A (TSA), Valproic acid (VPA), PXD101) were also tested. These also enhanced MICA/B surface levels in many of the cell lines, occasionally to even greater levels than doxycycline (Ovcar4, MCF-7) as well as in H596 cells where neither MMPi nor doxycycline had any effect. However, the increased surface expression levels of MICA/B after HDACi treatment also appeared to come with the cost of increased shedding in some cases (Figure 1C), indicating that the increased MICA/B levels after $\mathrm{HDACi}$ treatment may not translate into increased sensitivity to NKG2D expressing immune cells.

Doxycycline treatment increases overall MICA/B levels, movement to the surface and level of phosphorylation of ATM

In initial studies to help define the mechanisms driving doxycycline-mediated increases in MICA/B surface expression, two cell lines were examined, one that increased MICA/B surface expression in response to both MMPi and doxycycline (UCl-101) and one that responded to doxycycline only (Ovcar4). The overall levels of MICA/B in the cells were determined after different
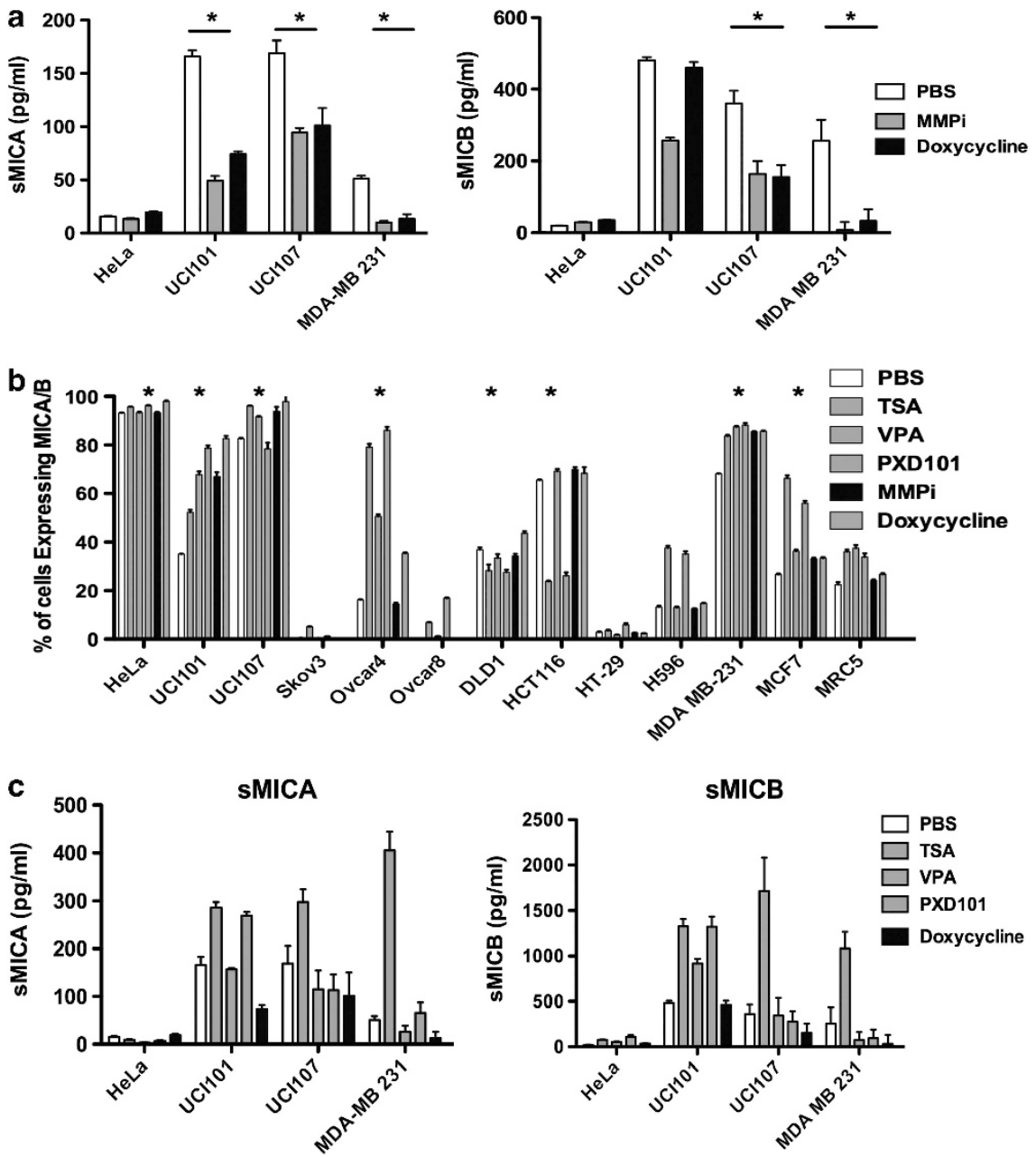

Figure 1. Doxycycline reduces shedding and enhances surface expression of MICA/B on tumor cells. (a) Doxycycline treatment reduces the shedding of soluble MICA (sMICA) or MICB (sMICB) from several cell lines. Doxycycline $\left(1 \mu \mathrm{g} \mathrm{ml}^{-1}\right)$ or MMPi III (5 $\left.\mu \mathrm{M}\right)$ were applied for $24 \mathrm{~h}$ and levels of soluble ligands in the media detected by ELISA after $24 \mathrm{~h}\left({ }^{*} P<0.05\right)$. (b) Surface expression levels of MICA/B after different treatments. Surface expression of MICA/B was determined by flow cytometry (using an antibody that recognizes both forms) at $24 \mathrm{~h}$ after various treatments. The percentages of cells demonstrating positive staining relative to an isotype control are shown. Doxycycline and MMPi III treatment as before; HDACi were also applied for $24 \mathrm{~h}$ (TSA was used at $0.375 \mu \mathrm{m}$; VPA was used at $750 \mu \mathrm{m}$; PXD101 was used at $0.625 \mu \mathrm{m}$ ) $\left({ }^{*} P<0.05\right)$. (c) Shedding of sMICA and SMICB was determined for cell lines after treatment with different HDACi (treatments and ELISA as before). 
a

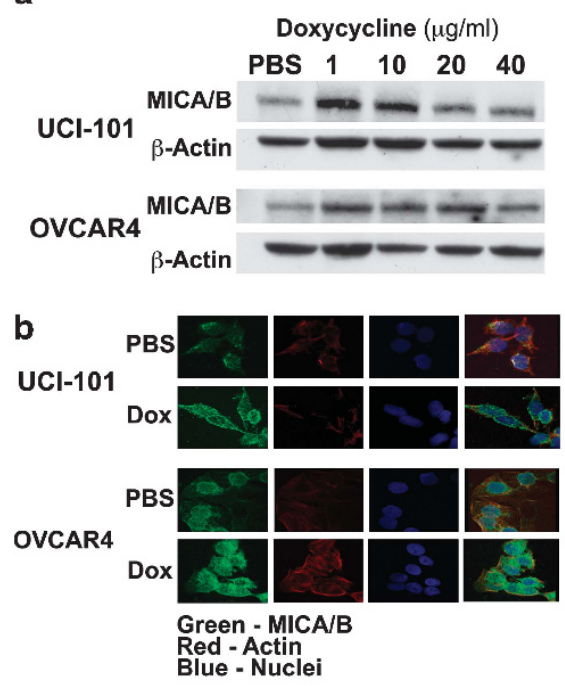

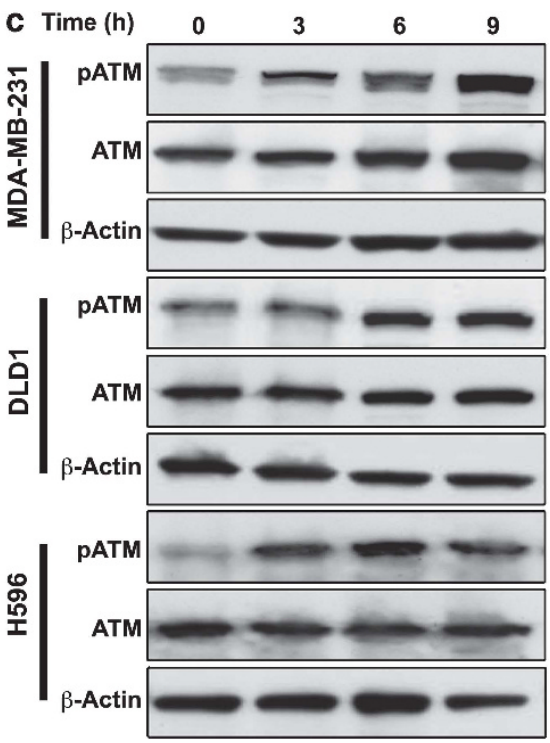

Figure 2. Doxycycline treatment enhances protein level and surface translocation of MICA/MICB, and enhances phosphorylated ATM levels. (a) Overall protein levels of MICA/B was detected in UCI-101 and OVCAR4 cell lines $24 \mathrm{~h}$ after treatment with the indicated concentrations of doxycycline by Western Blotting. (b) Immunofluorescence confocal microscopy was used to detect the subcellular location of MICA/B (green) after treatment with $40 \mu \mathrm{g} \mathrm{ml}^{-1}$ doxycycline for $24 \mathrm{~h}$ in UCl-101 and OVCAR4 cell lines. Phalloidin is red and Draq-5 (nuclei) are blue. $40 \times$ magnification. (c) Levels of phosphorylated ATM and overall ATM was determined in the indicated cell lines at the indicated times after treatment with $1 \mu \mathrm{g} \mathrm{ml}^{-1}$ doxycycline, as detected by western Blotting.

treatments by western blotting. In both cell lines the overall level of MICA/B within the cell was increased after doxycycline treatment (Figure 2a), while the movement of the MICA/B to the surface also appeared to be increased as determined by confocal microscopy (Figure 2b).

Although many of the factors influencing the surface expression levels of MICA/B are only poorly understood, most act through increasing transcription of the NKG2D ligands (e.g. heat shock, oxidative stress, HDACi). ${ }^{30}$ The only factor known to act both at a translational and post-translational level is ATM/Ataxia telangiectasia and Rad3 related (ATR) activation. ${ }^{31}$ The levels of phosphorylated ATM (pATM) were therefore examined by Western blotting in cell lines that responded to doxycycline and $\mathrm{MMPi}$ (MDA-MB-231), doxycycline only (DLD1) or neither (H596) (Figure 2c). In all these cell lines pATM levels increased, indicating that activation of ATM may mediate increased MICA/B levels in at least some cell lines treated with doxycycline. Doxycycline is known to have pleiotropic effects on treated cells in addition to MMPi properties, including scavenging of reactive oxygen species (ROS) $)^{32}$ and anti-apoptotic effects. ${ }^{33}$ Because ROS increase MICA/B transcription, scavenging of ROS is unlikely to cause increased surface expression. Anti-apoptotic effects may however influence pATM levels through the accumulation of DNA breaks, ${ }^{34}$ especially in tumor cells where DNA repair pathways are often defunct. However, no observable change in the level of apoptotic cells was observed after doxycycline treatment (data not shown).

Doxycycline treatment enhances sensitivity of tumor cells to killing by CIK cells in vitro and in vivo

CIK (Cytokine Induced Killer) cells, like many NK and some T cells, target NKG2D ligands including MICA/B on tumor targets. The capability of these cells to destroy tumor cells with and without doxycycline treatment was therefore examined in a simple in vitro CTL assay. Initially it was necessary to ensure that doxycycline treatment alone did not mediate tumor cell killing. None of the tumor cells examined was destroyed directly by doxycycline treatment (Figure 3a). However, all of the cell lines tested displayed increased sensitivity to CIK cells after even low dose doxycycline treatment $\left(1 \mu \mathrm{g} \mathrm{ml}^{-1}\right)$ (Figure 3b). This was apparently due to increased surface MICA/B expression as no increased killing was seen for SKOV3 cells that did not display enhanced MICA/B expression after doxycycline treatment.

In order to determine if the effects of doxycycline treatment could translate into enhanced anti-tumor effects in vivo a mouse tumor xenograft model was examined. UCl-101 cells were implanted subcutaneously into athymic nu-/nu- mice and when palpable $\left(50-100 \mathrm{~mm}^{3}\right)$ doxycycline $(100 \mu \mathrm{m})$ was added to the drinking water for $72 \mathrm{~h}$ prior to systemic treatment (tail vein) with $1 \times 10^{7}$ human CIK cells pre-labeled with Cy5.5 for fluorescence imaging of cell trafficking (mice remained on doxycycline treatment for a further 14 days after treatment). It was seen (Figure 4a) that CIK cells displayed significantly enhanced trafficking or targeting of tumors in mice that had been pretreated with doxycycline. This also translated into a significantly increased overall anti-tumor effect (Figure 4b). Doxycycline pretreatment can therefore enhance sensitivity to systemic treatment with CIK cells. No treatment-associated toxicities were observed with or without doxycycline treatment.

Doxycycline enhances oncolytic vaccinia replication in tumor cells We have previously described a novel therapeutic approach incorporating immune cells, such as CIK cells as carrier vehicles to deliver an oncolytic vaccinia virus to tumor targets after preinfection of the CIK cells with the virus. ${ }^{8}$ The effect of doxycycline exposure on vaccinia replication was therefore determined for strain vvDD (vaccinia containing deletions in the viral growth factor and thymidine kinase genes ${ }^{12,35}$ and expressing luciferase to quantify viral gene expression) (Figure 5).

Surprisingly doxycycline treatment increased viral replication in the majority of the tumor cell lines tested ( 8 of 12), this is of interest as several groups have described the incorporation of tetracycline responsive elements into vaccinia vectors, requiring doxycycline to induce or repress gene expression. ${ }^{36,37}$ Several non-tumor cell types were also tested (MRC-5 and CIK cells), and 


\section{a}
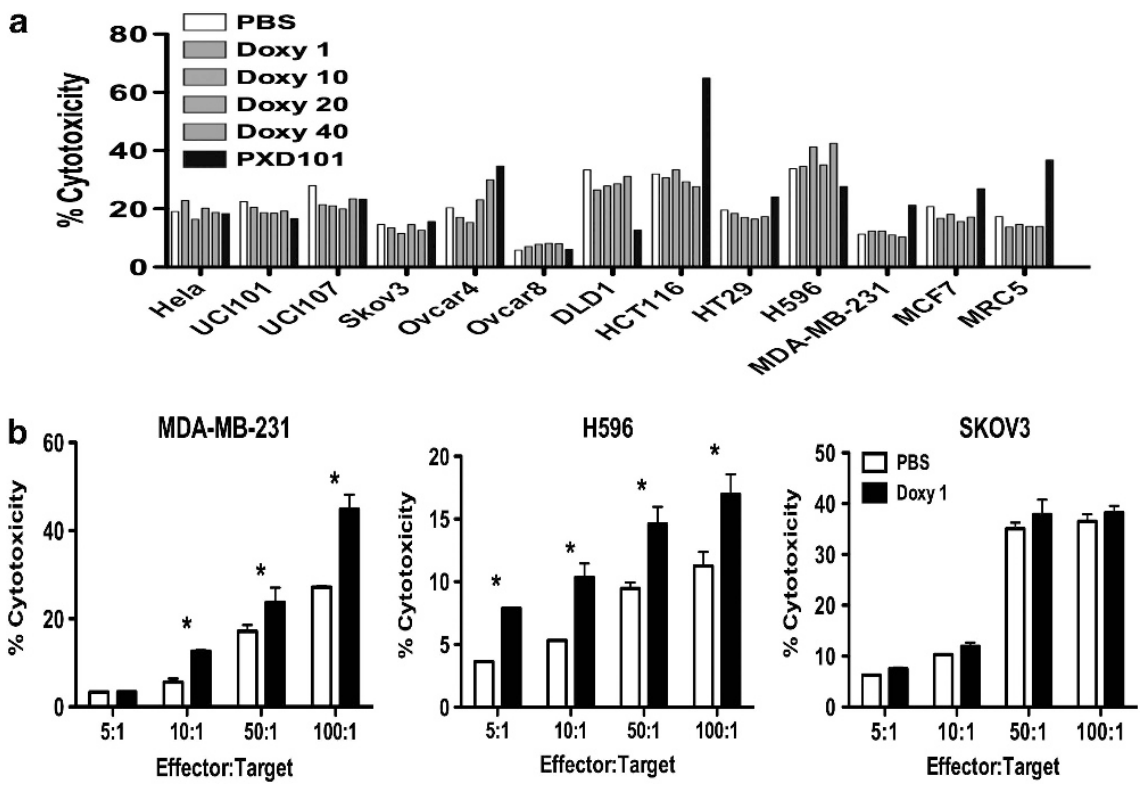

Figure 3. Doxycycline treatment enhances cytolytic capacity of CIK cells. (a) Initial tests determined the cytotoxicity of different treatments against different cell lines. Cells were treated with doxycycline $\left(1 ; 10 ; 20\right.$ or $\left.40 \mu \mathrm{g} \mathrm{ml}^{-1}\right)$ or PXD101 $(0.625 \mu \mathrm{M})$ for $24 \mathrm{~h}$ before. Cytotoxicity was determined as level of lactate dehydrogenase released into the medium relative to media alone (0\% cytotoxicity), or lysed cells (100\%). Doxycycline treatment did not result in significantly increased cytotoxicity $\left({ }^{*} P<0.05\right)$ for any cell lines or concentrations tested). (b) CIK mediated killing of selected cell lines. Human CIK cells ( $\mathrm{hCIK}$ ) were mixed with the indicated target tumor cells at the indicated effector:target ratios (between 5:1 and 100:1) which had or had not been treated with $1 \mu \mathrm{g} \mathrm{ml}^{-1}$ doxycycline for $24 \mathrm{~h}$ prior to the assay. Target cell survival was determined after $4 \mathrm{~h}$ by LDH assay as before.

doxycycline treatment did not appear to enhance viral replication in these cells. Because doxycycline enhances viral replication in a tumor specific manner, and did not inhibit viral replication in any cells, it is likely that both oncolytic virus therapy and CIK-VV combination therapy would be enhanced by pre-treatment or combined treatment with the antibiotic.

Although there were no clear patterns between cells that responded to doxycycline with respect to enhanced MICA/B surface expression and viral replication, it was noted that of the 4 cell lines that did not show increased viral replication, two (SKOV3 and Ovcar4) also did not increase MICA/B expression in response to doxycycline, while a third (MCF-7) only increased expression by a small degree.

In order to examine the effects of doxycycline treatment on the oncolytic viral therapy in vivo, MDA-MB-231 cells were implanted into mice and treated systemically (tail vein) with $1 \times 10^{7}$ PFU of oncolytic vaccinia virus strain vvDD expressing luciferase once tumors reached $50-100 \mathrm{~mm}^{3}$. Bioluminescence imaging was used to quantify viral gene expression from within the tumor at times after treatment for mice with and without additional doxycycline treatment. It was seen (Figure 6a) that doxycycline treatment significantly increased the level of viral gene expression from within the tumors of these animals. However, no such increase in viral gene expression was seen in non-tumor tissues, indicating that safety is not compromised with doxycycline treatment, instead an enhanced therapeutic index was achieved.

This increase in viral replication within the tumor also translated into a significantly increased anti-tumor effect in the same mouse model (Figure $6 \mathrm{~b}$ ). Doxycycline therefore has the capacity to enhance either CIK and oncolytic vaccinia therapies.

Doxycycline treatment significantly enhances CIK-VV therapy through action on multiple levels

The positive effect of doxycycline treatment on the action of both CIK cell and oncolytic vaccinia viral therapies used individually raised the expectation that even further enhanced therapeutic effects would also be seen when doxycycline is combined with CIK-VV therapy.

This was tested in vivo using MDA-MB-231 tumors implanted into athymic nu-/nu-mice. It was confirmed that the level of both CIK cell trafficking to the tumor (Figure $6 \mathrm{c}$ ) and the level of viral replication within the tumor (Figure 6d) were increased significantly when doxycycline was applied. This again translated into dramatically increased therapeutic benefit (Figure 6e). Whereas doxycycline alone had no effect on tumor growth, CIK-VV treatment resulted in significant responses, and 2 of 8 mice displayed complete and durable responses with a single intravenous injection of $1 \times 10^{7} \mathrm{CIK}$ cells pre-mixed with $1 \times$ $10^{7} \mathrm{PFU}$ of vVDD. The addition of doxycycline to the CIK-VV treatment however resulted in 6 of 8 durable complete responses.

\section{DISCUSSION}

Cytokine Induced Killer (CIK) cells have demonstrated encouraging clinical results in both the US $^{5}$ and Europe, ${ }^{38}$ and are routinely used in thousands of patients every year in China. They are one of a growing number of immune cell therapies that recognize stress response ligands such as MICA and MICB on the surface of cancer cells through binding to NKG2D. However many cancer have developed approaches to evade NKG2D mediated recognition, including shedding of soluble portions of MICA and MICB, ${ }^{21}$ reduction in the expression of these ligands or their internalization. ${ }^{23}$ We previously demonstrated that cell surface expression of MICA/B could be enhanced through application of Histone deacetylase inhibitors, leading to increased targeting and sensitivity to CIK therapy. ${ }^{24}$ However, this also frequently resulted in increased shedding of the $s M I C A / B$. One approach that blocked MICA/B shedding is to inhibit matrix metalloproteinase activity. ${ }^{22}$ However this approach is limited clinically by the lack of approved MMPi. Tetracyclines such as doxycycline, typically used as antibiotics, are known to have pleiotropic functions, including 
a
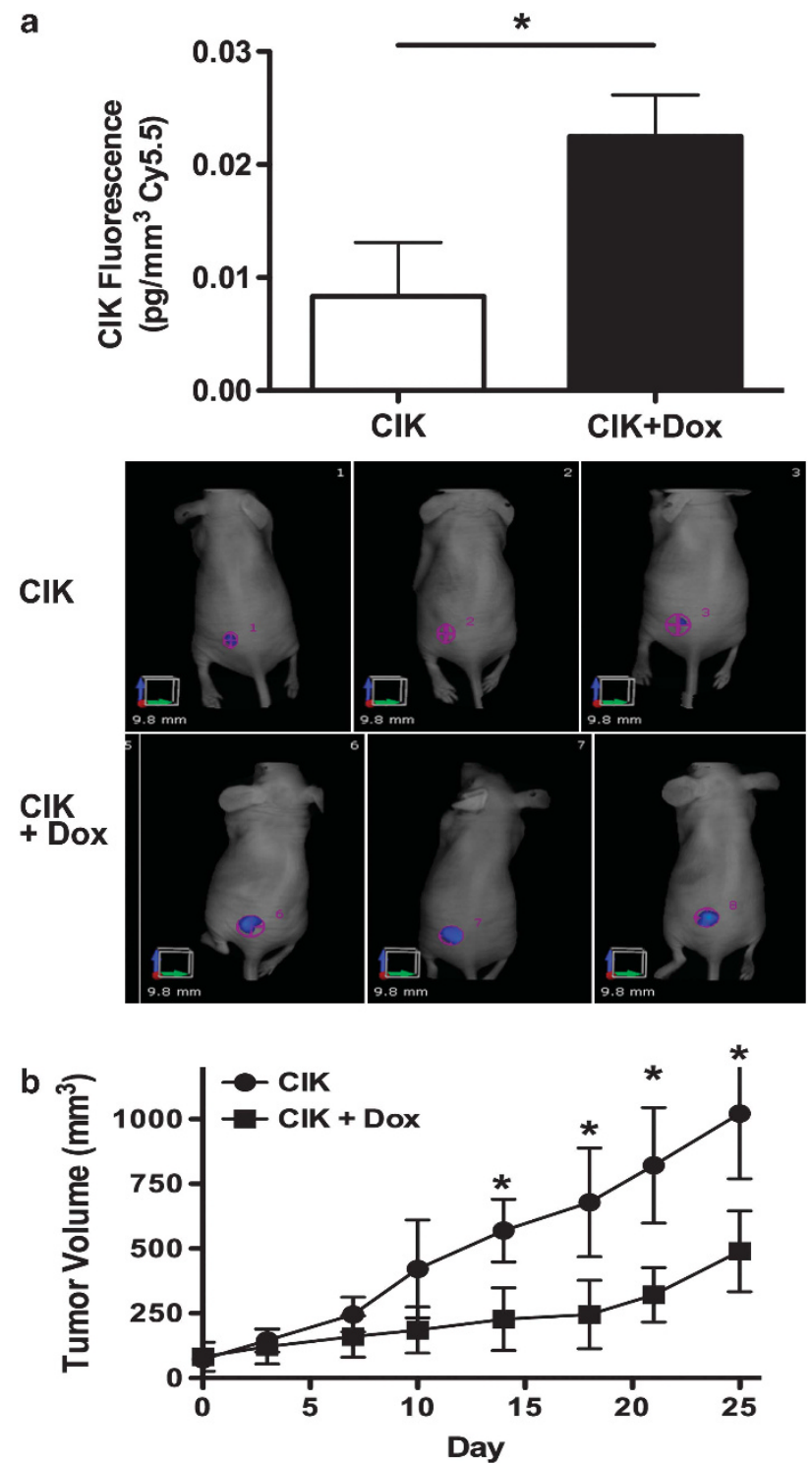

Figure 4. Doxycycline treatment enhances tumor targeting and therapeutic effect of CIK cells in mouse tumor models. (a) Athymic nu-/nu- mice were implanted with UCl-101 tumor cells $\left(1 \times 10^{7}\right.$ cells subcutaneously) and when tumors reached $50-100 \mathrm{~mm}^{3}$ treatments were begun. Some mice received doxycycline in their drinking water $(100 \mu \mathrm{m})$ for a total of 17 days starting 3 days before CIK cell treatment. Human CIK cells (hCIK) were pre-labeled with cy5.5 before $1 \times 10^{7}$ cells were delivered via tail vein injection. The number of CIK cells in the tumor was determined by whole animal fluorescence imaging $72 \mathrm{~h}$ after initial injection. Significantly more CIK cell fluorescence was detected in the tumors of doxycycline treated mice $\left(n=8\right.$ per group; $\left.{ }^{*} P=0.03\right)$. Sample images are shown. (b) Anti-tumor effects of CIK cells used as before with and without doxycycline combination. The tumor growth and response to therapy were followed in the same mice by caliper measurement. Doxycycline enhanced the anti-tumor effects of CIK cell therapy ( ${ }^{*}<<0.05$ for days $\left.14-24\right)$.

MMPi function. ${ }^{28}$ Our initial aim was therefore to examine whether the MMPi function of doxycycline was capable of blocking MICA/B cleavage from the surface of cancer cells.

Surprisingly, doxycycline not only blocked cleavage and increased surface expression of MICA/B in cells known to produce sMICA/B, but also increased surface expression in cancer cells that did not shed the ligands, so negating any requirement for adding
HDACi (to upregulate expression) in addition to MMPi (to block shedding). This was unexpected, and greatly increases the potential utility of doxycycline as an adjuvant to immune cell therapies. Initial investigation into the mechanism underlying this effect determined that doxycycline apparently increased both the overall level and surface trafficking of MICA/B. Although much is still unknown about the regulation of cell surface MICA/B levels, activation of ATM/ATR is the only recognized mechanism that enhances both transcription and cell surface trafficking. ${ }^{31}$ The level of phosphorylated ATM after doxycycline treatment was therefore examined, and an increase was found in many cell lines. However it should be noted that pATM levels increased in both cell lines that responded to doxycycline treatment and those that did not, indicating additional suppressive mechanisms might also be involved.

It is also unclear how doxycycline increases PATM levels, however there may be a link between the known activation of ATM as a result of increased DNA damage, ${ }^{34}$ the increased levels of DNA damage in tumor cells, and the known anti-apoptotic effects of tetracyclines. ${ }^{33}$ Possibly inhibition of apoptosis may lead to increased accumulation of DNA damage, especially in tumor cells, and so increased levels of pATM and MICA/B surface expression, however this needs further testing.

It was evident that doxycycline treatment in mouse tumor models led to enhanced CIK targeting and greater antitumor effects. We have previously described a highly promising combination therapy in pre-clinical models involving the combination of CIK cells with an oncolytic vaccinia virus, ${ }^{8}$ such that the cells can be pre-infected with virus and act as carrier vehicles, releasing the virus selectively in the tumor. The virus in turn acts as means for arming the CIK cells, and greatly increasing their capacity to destroy the tumor once they infiltrate. It is reasonable to expect that doxycycline would therefore also act to enhance the power of this CIK-VV combination therapy. However it was necessary to initially verify that doxycycline did not inhibit viral replication in the tumor cells. Surprisingly doxycycline treatment actually enhanced viral replication in the majority of tumor cells examined. This unexpected result has additional implications for the incorporation of tetracycline response elements into vaccinia strains to follow the effects of selective viral gene expression. Although, the mechanism is not understood, it appears that doxycycline effects on pATM levels, blocking of apoptosis or scavenging of reactive oxidative species is not the primary cause.

However, this effect of doxycycline on viral replication might be used to enhance the effects of oncolytic vaccinia therapies both used alone or especially in combination with CIK cell carrier vehicles. Indeed, doxycycline treatment was seen to significantly increase both viral replication selectively in the tumor and overall therapeutic effect in mouse tumor models. Furthermore, the effects of doxycycline on the anti-tumor effects of CIK-VV therapy were even more dramatic with a highly significant therapeutic benefit.

The current routine use of CIK cells in treatment of cancers in some regions, and the promising clinical data seen in recent Western trials mean that approaches that further increase their effectiveness and the range of cancers they can effectively treat would be highly beneficial. In addition, the recent exciting clinical data with oncolytic vaccinia virus raises the likelihood that these therapies may soon be approved for use in Western markets. Again, the observation that combining oncolytic vaccinia with a commonly used and approved agent such as doxycycline can safely enhance its therapeutic benefit would be important. Finally although CIK-VV combination therapies have only been examined in pre-clinical models to date, the combination has displayed improved therapeutic benefit over either CIK or VV used as single agents and clinical development is ongoing. The dramatically improved therapeutic effects of combining all these therapies with doxycycline make this an exciting potential future combination 

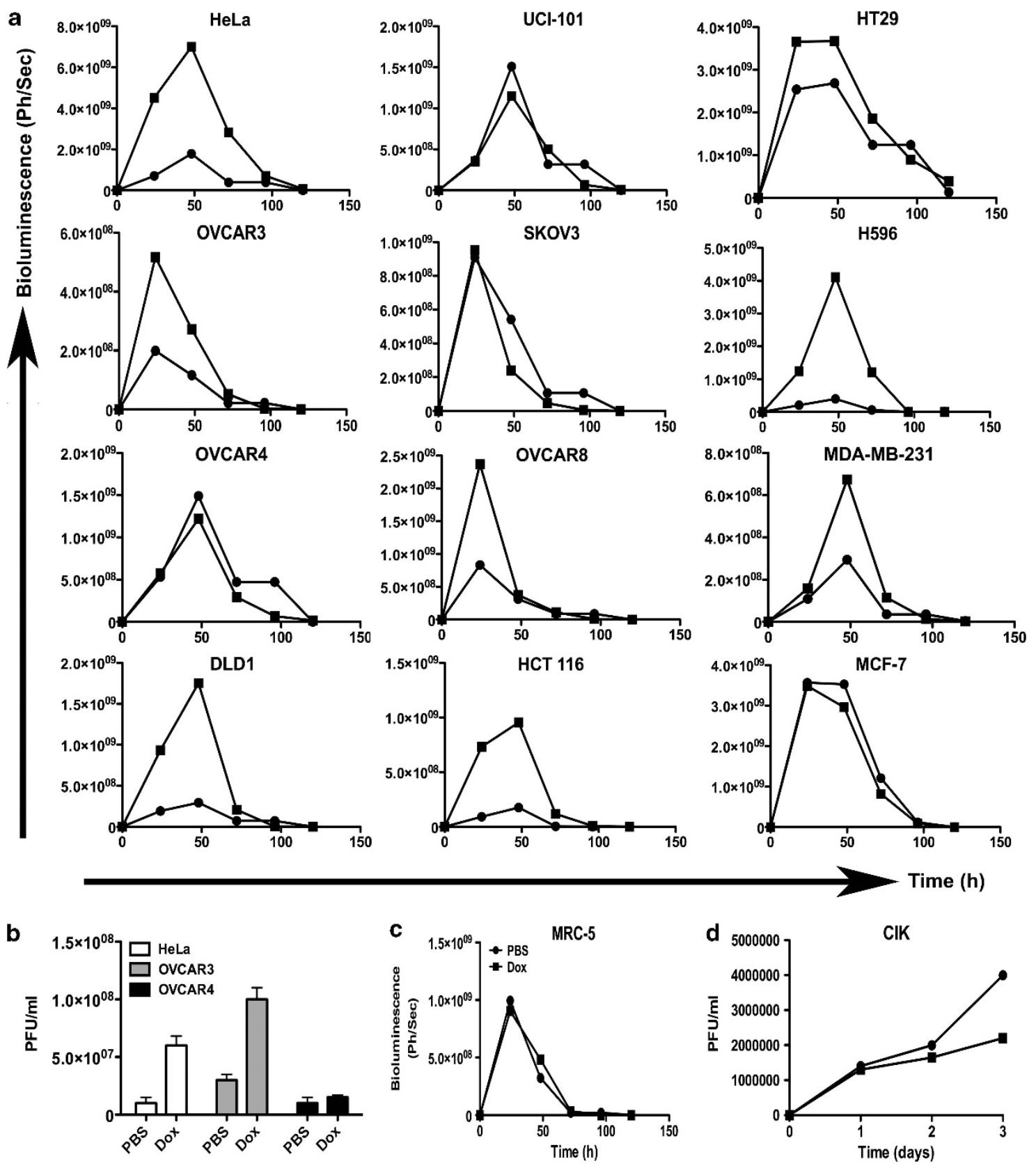

Figure 5. Doxycycline enhances viral replication and gene expression after treatment of multiple tumor cell lines with oncolytic vaccinia strain vvDD. (a) Different tumor cell lines were infected (Multiplicity of Infection of $1.0 \mathrm{PFU} / \mathrm{cell}$ ) with oncolytic vaccinia strain vvDD expressing luciferase, with and without the presence of $1 \mu \mathrm{gml}^{-1}$ doxycycline. Viral gene expression was determined at indicated times by bioluminescence imaging. Cell lines HeLa, HT29, OVCAR3, H596, OVCAR8, MDA-MB-231, DLD1 and HCT116 all displayed significantly increased gene expression at $48 \mathrm{~h}$ after infection, $(P<0.05)$. (b) Viral plaque assays confirmed the increased gene expression correlated with viral replication for selected cell lines at $48 \mathrm{~h}$. (c) The same gene expression assay was performed on the non-tumor (immortalized, non-transformed) cell line MRC-5. (d) Viral replication was also determined by plaque assay $r$ in human CIK cells.

approach. A variety of other immune cell based therapies also target NKG2D ligand expression on tumors and so these might also be enhanced by combination with doxycycline, while a number of other oncolytic virus strains are undergoing advanced clinical testing, and it would be of interest to know if they also benefit from doxycycline combination.

\section{MATERIALS AND METHODS}

Cell lines and reagents

Human ovarian cancer cell line UCl-101 and UCl-107 were a kind gift from Drs P DiSaia and A Menetta (University of California, San Diego). Ovcar4 and Ovcar8 were obtained from Dr T Hamilton (NCI-Frederick Cancer DCTD Tumor/Cell Line Repository, Frederick, MD, USA). Human ovarian cancer cell lines Skov3, cervical cancer cell line Hela, human colon cancer cell lines DLD1, HCT116 and HT29, human lung cancer cell line H596 and human normal lung fibroblast cell line MRC5 were obtained from American Type Culture Collection (ATCC), Manassas, VA, USA. All cells were cultured in recommended culture media supplemented with 5 or $10 \%$ fetal bovine serum and antibiotics.

Histone Dacetylase Inhibitors: TSA (Trichostatin A) and VPA (Valproic acid sodium salt) were purchased from Sigma-Aldrich (St Louis, MO, USA); Doxycycline was purchased from MP Biomedicals, Santa Ana, CA, USA; PXD101 (Belinostat) was obtained from Selleck Chemicals, Houston, TX, USA; The MMP inhibitor III was from Calbiochem (Merck, Darmstadt, 

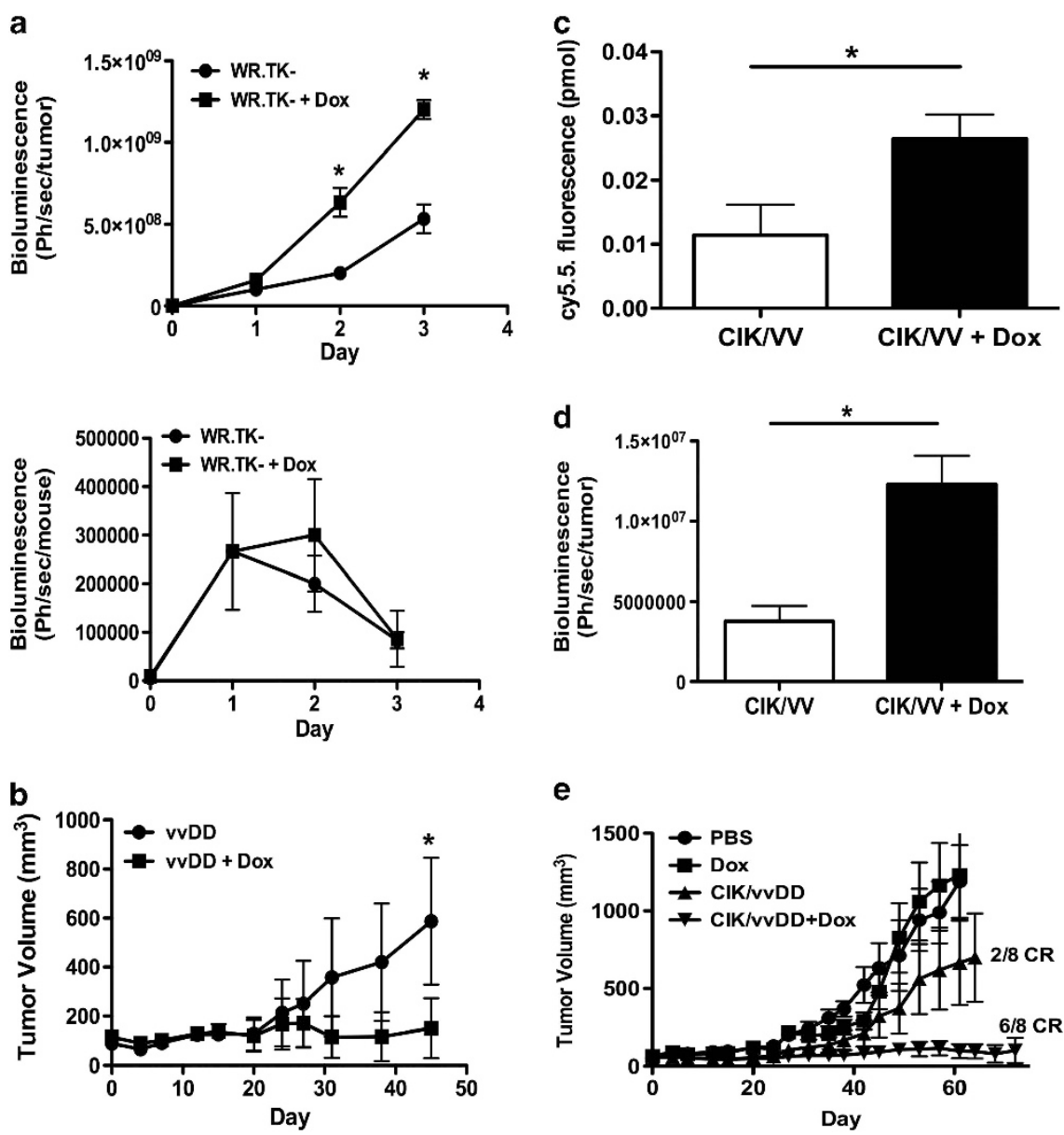

Figure 6. Doxycycline enhances in vivo effects of oncolytic vaccinia therapy alone, or in combination with CIK cell-mediated viral delivery. (a) Mice (athymic nu-/nu- bearing subcutaneous MDA-MB 231 tumors of $50-100 \mathrm{~mm}^{3}$ ) were treated with oncolytic vaccinia strain WR.TK- expressing luciferase $\left(1 \times 10^{7}\right.$ PFU delivered via tail vein) with or without doxycycline $(n=7$ per group). Subsequent viral gene expression was determined by bioluminescence imaging at the indicated times for a region of interest over the tumor (top) or the torso (bottom) of the mice $\left({ }^{*} P<0.05\right)$. (b) Therapeutic effect of the same treatments with tumor volume determined by caliper measurement $(* P<0.05)$. (c) Mice as before (athymic nu-/nu- with MDA MB 231 tumors) were treated with PBS, doxycycline, hCIK $\left(1 \times 10^{7}\right)$ pre-mixed with $\operatorname{vVDD}\left(1 \times 10^{7} \mathrm{PFU}\right)$; or CIK/vvDD and doxycycline $(n=10$ per group). CIK cells were labeled with cy5.5 prior to injection and fluorescence from within the tumor was determined after $72 \mathrm{~h}$ by whole animal fluorescence imaging $\left({ }^{*} P=0.027\right)$. (d) Bioluminescence imaging t the same time point determined viral gene expression from within the tumor $(* P=0.0009)$. (e) Caliper measurements followed subsequent effect on tumor volume. Doxycycline alone had no effect on tumor growth, whereas CIK/vvDD showed significant benefit relative to control from day 60 , and $\mathrm{CIK} / \mathrm{vvDD}$ plus doxycycline was significantly better than any other treatment from day 44 onwards $(P<0.5)$. At the end of the experiment 2 of 8 mice in the CIK-vVDD group displayed complete responses and 6 of 8 in the CIK/vvDD plus doxycycline group.

Germany). Unless otherwise stated TSA was used at $0.375 \mu$ m; VPA was used at $750 \mu \mathrm{m} ;$ PXD101 was used at $0.625 \mu \mathrm{m} ; \mathrm{MMP}$ inhibitor III was used at $5 \mu \mathrm{m}$; Doxycycline was used at concentrations range from 1 to $40 \mu \mathrm{g} \mathrm{ml}^{-1}$ for in vitro and $100 \mathrm{\mu g} \mathrm{ml}^{-1}$ in drinking water for in vivo work. The usual dose for treatment of infections in humans is $100 \mathrm{mg}$ twice a day oral or IV (depending on the pathogen), meaning that the doses used here are below typical doses on a per $\mathrm{kg}$ basis.

\section{Flow cytometry}

Cell surface MICA/MICB detection was with PE-conjugated anti-human MICA/MICB (eBioscience, San Diego, CA, USA). PE-conjugated mouse IgG ak isotype was used as control (eBioscience, San Diego, CA, USA). For cell apoptosis analysis FITC-conjugated Annexin-V and Propidium lodide (PI) was used according to the manufacturer's directions (Abcam Inc., Cambridge, MA). Samples were analyzed using a BD Accuri C6 Flow Cytometer (Becton, Dickinson and Company, Franklin Lakes, NJ, USA) and analysis on FlowJo (Treestar Inc, Ashland, OR, USA).

Cell immunofluorescence

Cells ( 5000 cells/chamber) were seeded and incubated overnight on 4-chambers chamber slides (Lab-Tek, Scott's Valley, CA, USA). After incubation with indicated doses of Doxycycline for $24 \mathrm{~h}$, cells were fixed with $2 \%$ paraformaldehyde and immunofluorescence was performed with mouse anti-human monoclonal to MICA + MICB (Abcam Inc., Cambridge, MA, USA), followed by the appropriate secondary antibody conjugated to Alexa Fluor 488 (Invitrogen, Carlsbad, CA, USA) at a dilution of 1:600. Alexa Fluor 546- conjugated phalloidin was used at a 1:1000 dilution. To visualize nuclei, slides were incubated for $10 \mathrm{~min}$ in DRAQ-5 (Biostatus Limited, Shepshed, UK) diluted to 1:1000 in TBS (20 mM Tris-HCl, pH 7.5, $500 \mathrm{~mm}$ $\mathrm{NaCl}$ ). Fluorescent images were collected using a Leica TCS SL Confocal microscope (Leica Microsystems, Bannockburn, IL, USA).

\section{Western blot analysis}

Protein extracts were isolated from $10^{6}$ treated cells using the mammalian cell lysis reagent containing protease inhibitor cocktail (Sigma-Aldrich, St Louis, MO, USA) following the manufacturer's directions. Equal amounts of lysate protein were resolved on a $4-20 \%$ precast polyacrylamide gel (Bio-Rad Laboratories, Inc., Hercules, CA, USA) and were transferred to Immobilon-P polyvinylidene difluoride membrane (Millipore, Billerica, MA, USA). MICA/B, Ataxia telangiectasia mutated kinase (ATM), Phospho-ATM and $\beta$-actin proteins were detected on Western blots using the mouse monoclonal to MICA + MICB (Abcam Inc., Cambridge, MA, USA), ATM (D2E2) Rabbit mAb, Phospho-ATM (Ser1981) (D6H9) Rabbit mAb (Cell 
Signaling Technology, Inc., Danvers, MA, USA), mouse monoclonal to $\beta$-actin (Santa Cruz Biotechnology, Inc., Santa Cruz, CA, USA) antibodies, respectively. Blots were then incubated with appropriate peroxidaseconjugated secondary antibodies, and detected using the SuperSignal chemiluminescence system (Thermo Fisher Scientific, Rockville, MD, USA).

\section{ELISA}

ELISA measurement of soluble MICA and MICB levels in cell culture medium were determined by Human MICA Duoset ELISA Development kit and Human MICB Duoset ELISA Development kit (R\&D Systems, Inc. Minneapolis, MN, USA). The procedures are in accordance with the protocols supplied with the kit.

\section{Cellular cytotoxicity assay}

Cell cytotoxicity was evaluated using a CytoTox 96 non-radioactive cytotoxicity assay kit (Promega, Madison, WI, USA) based on the measurement of lactase dehydrogenase (LDH) according to the manufacturer's instructions. For CIK cell-mediated cytotoxicity assays, hCIK cells were prepared as stated before and were added to tumor cells using effector to target cell ratios of 50:1,20:1, 10:1, and 1:1. After a $4 \mathrm{~h}$ incubation at $37^{\circ} \mathrm{C}$, culture medium was harvested for LDH production analysis, according to the manufacturer's instructions.

\section{Viral infection}

Cell lines were treated as indicated for $24 \mathrm{~h}$, then challenged with luciferase (Luc)- expressing oncolytic vaccinia virus strains WR.TK-, with a single deletion in the viral thymidine kinase gene $(T K)$, and VvDD, with deletions in both the TK and vaccinia growth factor (VGF) genes, at a multiplicity of infection (MOI) of 1 plaque forming units (pfu) per cell. At indicated time points post-infection, luciferin was added to each well $[10 \mu \mathrm{l} /$ well of $30 \mathrm{mg} / \mathrm{ml}$ luciferin (Caliper Life Science, Perkin Elmer, Waltham, MA, USA)] and bioluminescence per well (photons/second) was measured on an IVIS 200 imaging system (Xenogen part of Perkin Elmer). In some experiments viral replication was determined by plaque assay on BSC-1 cell layers. All experiments were run in triplicate.

\section{Mouse models}

Athymic nu-/nu- mice (female, 6-8 weeks) obtained from Taconic Corporation (Germantown, NY, USA) were used for xenograft studies. Mice received subcutaneous injection of $1.5 \times 10^{7} \mathrm{UCl}-101$ or MDA MB 231 tumor cells. Once palpable tumors $\left(50-100 \mathrm{~mm}^{3}\right)$ were formed, animals were regrouped and treatment was begun. Mice were treated with Doxycycline ( $100 \mu \mathrm{m}$ in drink water) 3 days prior to injection of CIK cells, virus or virus premixed with $\mathrm{hClK}$ cells, and up to 14 days after treatment. $1 \times 10^{7} \mathrm{hCIK}$ cells were premixed for $14 \mathrm{~h}$ with $1 \times 10^{7} \mathrm{PFU}$ of vVDD, the hCIK cells were labeled using cy5.5 NHS ester (Lumiprobe Corporation, Hallandale, FL, USA) half an hour before injection, and delivered via intravenous tail vein injection. Subsequent tumor volumes were determined by caliper measurement (volume $=$ length $\times$ width $^{2} \times \pi / 6$ ) and animals euthanized when tumors reached $1.4 \mathrm{~cm}^{3}$. All animal studies were approved by Institutional Animal Care and Use Committee (IACUC), University of Pittsburgh.

Mice treated with luciferase-expressing virus were imaged using an IVIS 200 imaging system (Xenogen; Caliper Life Sciences), Mice were injected i.p. with luciferin $(30 \mathrm{mg} / \mathrm{kg}$ ) and anesthetized ( $2 \%$ isoflurane) prior to imaging. Cy 5.5 labeled hCIK cell were imaged using the Fluorescence Molecular Tomography (FMT) 2500 system (Perkin Elmer).

\section{Statistics}

Student $t$-tests were used throughout, with statistical significance considered to be $P<0.05$.

\section{CONFLICT OF INTEREST}

Dr Thorne has a financial interest in Jennerex Biotherapeutics. The remaining authors declare no conflict of interest.

\section{ACKNOWLEDGEMENTS}

This work was supported by NCl NIH grant R01 CA140215 (SHT). UCl-101 and UCl-107 cells were a kind gift from Drs P DiSaia and A Menetta (University of California,
San Diego). Ovcar4 and Ovcar8 were obtained from Dr T Hamilton (NCI-Frederick Cancer DCTD Tumor/Cell Line Repository).

\section{REFERENCES}

1 Turcotte S, Rosenberg SA. Immunotherapy for metastatic solid cancers. Adv Surg 2011; 45: 341-360.

2 Rosenberg SA, Yang JC, Sherry RM, Kammula US, Hughes MS, Phan GQ et al. Durable complete responses in heavily pretreated patients with metastatic melanoma using T-cell transfer immunotherapy. Clinical Cancer Research: an Official Journal of the American Association for Cancer Research 2011; 17: 4550-4557.

3 Robbins PF, Morgan RA, Feldman SA, Yang JC, Sherry RM, Dudley ME et al. Tumor regression in patients with metastatic synovial cell sarcoma and melanoma using genetically engineered lymphocytes reactive with NY-ESO-1. Journal of Clinical Oncology: Official Journal of the American Society of Clinical Oncology 2011; 29: 917-924.

4 Geller MA, Miller JS. Use of allogeneic NK cells for cancer immunotherapy. Immunotherapy 2011; 3: 1445-1459.

5 Leemhuis T, Wells S, Scheffold C, Edinger M, Negrin RS. A phase I trial of autologous cytokine-induced killer cells for the treatment of relapsed Hodgkin disease and non-Hodgkin lymphoma. Biol Blood Marrow Transplant 2005; 11: 181-187.

6 Schmidt-Wolf IG, Lefterova P, Mehta BA, Fernandez LP, Huhn D, Blume KG et al. Phenotypic characterization and identification of effector cells involved in tumor cell recognition of cytokine-induced killer cells. Exp Hematol 1993; 21: 1673-1679.

7 Lu PH, Negrin RS. A novel population of expanded human CD3 + CD56 + cells derived from $T$ cells with potent in vivo antitumor activity in mice with severe combined immunodeficiency. J Immunol 1994; 153: 1687-1696.

8 Thorne SH, Negrin RS, Contag $\mathrm{CH}$. Synergistic antitumor effects of immune cell-viral biotherapy. Science 2006; 311: 1780-1784.

9 Contag CH, Sikorski R, Negrin RS, Schmidt T, Fan AC, Bachireddy P et al. Definition of an enhanced immune cell therapy in mice that can target stem-like lymphoma cells. Cancer Res 2010; 70: 9837-9845.

10 Guo ZS, Thorne SH, Bartlett DL. Oncolytic virotherapy: molecular targets in tumorselective replication and carrier cell-mediated delivery of oncolytic viruses. Biochim Biophys Acta 2008; 1785: 217-231.

11 Kirn DH, Wang Y, Le Boeuf F, Bell J, Thorne SH. Targeting of interferon-beta to produce a specific, multi-mechanistic oncolytic vaccinia virus. PLoS Med 2007; 4: e353.

12 Thorne SH, Hwang TH, O'Gorman WE, Bartlett DL, Sei S, Kanji F et al. Rational strain selection and engineering creates a broad-spectrum, systemically effective oncolytic poxvirus, JX-963. J Clin Invest 2007; 117: 3350-3358.

13 Park BH, Hwang T, Liu TC, Sze DY, Kim JS, Kwon HC et al. Use of a targeted oncolytic poxvirus, JX-594, in patients with refractory primary or metastatic liver cancer: a phase I trial. Lancet Oncol 2008; 9: 533-542.

14 Kirn DH, Thorne SH. Targeted and armed oncolytic poxviruses: a novel multi-mechanistic therapeutic class for cancer. Nat Rev Cancer 2009; 9: 64-71.

15 Breitbach CJ, Burke J, Jonker D, Stephenson J, Haas AR, Chow LQ et al. Intravenous delivery of a multi-mechanistic cancer-targeted oncolytic poxvirus in humans. Nature 2011; 477: 99-102.

16 Melder RJ, Whiteside TL, Vujanovic NL, Hiserodt JC, Herberman RB. A new approach to generating antitumor effectors for adoptive immunotherapy using human adherent lymphokine-activated killer cells. Cancer Res 1988; 48: 3461-3469.

17 Sasaki A, Melder RJ, Whiteside TL, Herberman RB, Jain RK. Preferential localization of human adherent lymphokine-activated killer cells in tumor microcirculation. J Natl Cancer Inst 1991; 83: 433-437.

18 Visonneau S, Cesano A, Porter DL, Luger SL, Schuchter L, Kamoun M et al. Phase trial of TALL-104 cells in patients with refractory metastatic breast cancer. Clin Cancer Res 2000; 6: 1744-1754.

19 Tonn T, Becker S, Esser R, Schwabe D, Seifried E. Cellular immunotherapy of malignancies using the clonal natural killer cell line NK-92. J Hematother Stem Cell Res 2001; 10: 535-544.

20 Bauer S, Groh V, Wu J, Steinle A, Phillips JH, Lanier LL et al. Activation of NK cells and T cells by NKG2D, a receptor for stress-inducible MICA. Science 1999; 285: 727-729.

21 Groh V, Wu J, Yee C, Spies T. Tumour-derived soluble MIC ligands impair expression of NKG2D and T-cell activation. Nature 2002; 419: 734-738.

22 Kaiser BK, Yim D, Chow IT, Gonzalez S, Dai Z, Mann HH et al. Disulphideisomerase-enabled shedding of tumour-associated NKG2D ligands. Nature 2007; 447: 482-486.

23 Long EO. Tumor cell recognition by natural killer cells. Semin Cancer Biol 2002; 12: 57-61.

24 Huang B, Sikorski R, Sampath P, Thorne SH. Modulation of NKG2D-ligand cell surface expression enhances immune cell therapy of cancer. $J$ Immunother 2011; 34: 289-296. 
25 Armeanu S, Bitzer M, Lauer UM, Venturelli S, Pathil A, Krusch M et al. Natural killer cell-mediated lysis of hepatoma cells via specific induction of NKG2D ligands by the histone deacetylase inhibitor sodium valproate. Cancer Res 2005; 65 6321-6329.

26 Skov S, Pedersen MT, Andresen L, Straten PT, Woetmann A, Odum N. Cancer cells become susceptible to natural killer cell killing after exposure to histone deacetylase inhibitors due to glycogen synthase kinase-3-dependent expression of MHC class I-related chain A and B. Cancer Res 2005; 65: 11136-11145.

27 Peterson JT. Matrix metalloproteinase inhibitor development and the remodeling of drug discovery. Heart Fail Rev 2004; 9: 63-79.

28 Burns FR, Stack MS, Gray RD, Paterson CA. Inhibition of purified collagenase from alkali-burned rabbit corneas. Invest Ophthalmol Vis Sci 1989; 30: 1569-1575.

29 Griffin MO, Fricovsky E, Ceballos G, Villarreal F. Tetracyclines: a pleitropic family of compounds with promising therapeutic properties. Review of the literature. Am J Physiol Cell Physiol 2010; 299: C539-C548.

30 Mistry AR, O'Callaghan CA. Regulation of ligands for the activating receptor NKG2D. Immunology 2007; 121: 439-447.

31 Gasser S, Orsulic S, Brown EJ, Raulet DH. The DNA damage pathway regulates innate immune system ligands of the NKG2D receptor. Nature 2005; 436: 1186-1190.
32 Kraus RL, Pasieczny R, Lariosa-Willingham K, Turner MS, Jiang A, Trauger JW. Antioxidant properties of minocycline: neuroprotection in an oxidative stress assay and direct radical-scavenging activity. J Neurochem 2005; 94: 819-827.

33 Yrjanheikki J, Keinanen R, Pellikka M, Hokfelt T, Koistinaho J. Tetracyclines inhibit microglial activation and are neuroprotective in global brain ischemia. Proc Nat Acad Sci USA 1998; 95: 15769-15774.

34 Bakkenist CJ, Kastan MB. DNA damage activates ATM through intermolecular autophosphorylation and dimer dissociation. Nature 2003; 421: 499-506.

35 McCart JA, Ward JM, Lee J, Hu Y, Alexander HR, Libutti SK et al. Systemic cancer therapy with a tumor-selective vaccinia virus mutant lacking thymidine kinase and vaccinia growth factor genes. Cancer Res 2001; 61: 8751-8757.

36 Traktman P, Liu K, DeMasi J, Rollins R, Jesty S, Unger B. Elucidating the essential role of the A14 phosphoprotein in vaccinia virus morphogenesis: construction and characterization of a tetracycline-inducible recombinant. J Virol 2000; 74: 3682-3695.

37 Hedengren-Olcott $M$, Hruby DE. Conditional expression of vaccinia virus genes in mammalian cell lines expressing the tetracycline repressor. J Virol Methods 2004 120: 9-12.

38 Rutella S, ludicone P, Bonanno G, Fioravanti D, Procoli A, Lavorino C et al. Adoptive immunotherapy with cytokine-induced killer cells generated with a new good manufacturing practice-grade protocol. Cytotherapy 2012; 14: 841-850. 